\title{
Should Tenofovir Ever be Used in Association with Didanosine?
}

\section{Ricardo Sobhie Diaz}

Retrovirology Laboratory, Medicine School of São Paulo; São Paulo, SP, Brazil
Tenofovir disoproxil fumarate is a nucleotide analogue that is rapidly metabolized intracellularly to its active anabolite, tenofovir diphosphate, which is a competitive inhibitor of HIV-1 reverse transcriptase; it terminates the elongation of the DNA chain. As the recommended oral dosage of tenofovir in adults is 300 mg given once daily, its use with other well-tolerated once-daily drugs rapidly became popular. However, this habit has resulted in unexpectedly high rates of virologic failures. In one study, in which once a day tenofovir, lamivudine and abacavir was used for 12 months in 36 naive patients [1], virological failure was observed in 12 of the them. Among these 12 patients, 11 had both K65R and M184V mutations and one had the M184V mutation alone. While K65R is a resistance mutation for tenofovir, $\mathrm{M} 184 \mathrm{~V}$ is a resistance mutation for lamivudine, and both are resistance mutations for abacavir. Low genetic barriers to resistance was considered the main cause of failure, since only two HIV mutations were enough to impair this triple antiretroviral association. In another 24-week pilot study, the rate of virologic failure of once a day tenofovir/ddI(didanosine)/lamivudine in naive patients was even higher [2]. Of 22 patients, 20 (91\%) discontinued treatment due to a suboptimal response. Resistance testing showed M184I/V in all patients, with 10 of these also having K65R. Another recent randomized controlled trial compared tenofovir/ddI/ efavirenz, with or without Lopinavir/ritonavir, for 12 weeks in naive patients [3]. Six out of 14 patients

Received on 10 August; revised 19 October 2005.

Address for correspondence: Dr. Ricardo Sobhie Diaz. Retrovirology Laboratory, Paulista School of Medicine, Federal University of São Paulo. R. Pedro de Toledo, 781, São Paulo, SP-04039 - Brazil.Email: rsdiaz@usp.br.

The Brazilian Journal of Infectious Diseases 2005;9(6):452-453 (C) 2005 by The Brazilian Journal of Infectious Diseases and Contexto Publishing. All rights reserved. receiving tenofovir/ddI/efavirenz alone developed early virologic failure and drug resistance, whereas none of the 12 patients in the lopinavir arm experienced virologic failure. Mutations L74V/I (ddI) and K65R (ddI and tenofovir) were present in four and two patients respectively, and G190S/E and K103N (both efavirenz) were present in five and one patient respectively. All failures had baseline HIV-1 RNA levels above 100,000 copies/mL.

In contrast to what is seen for regimens containing tenofovir/lamivudine/abacavir, the low genetic barrier for failure is probably not a good explanation for the tenofovir/ddI failures. The primary rationale for combination antiretroviral therapy is to inhibit different steps or substeps of viral replication, or to inhibit any given step in different ways. Unfortunately, both tenofovir and ddI are adenosine analogs and therefore block the same sub-step on the HIV replication cycle in an identical manner. One possibility is that the combination of tenofovir and ddI will work as if there were only one and not two drugs in the regimen scheme, making triple therapies really be double therapies. Tenofovir and ddI generally share the same antiretroviral resistance profile, and sometimes these two drugs are the only nucleoside/nucleotides analogs left to be used in more experienced patients. Given these observations, plus unclear pharmacokinetic interactions [4] and a possible lymphotoxic effect of these drugs, [5] the combination of tenofovir and ddI should be avoided.

\section{References}

1. Landman R., Peytavin G., Descamps D., et al. Low Genetic Barrier to Resistance Is a Possible Cause of Early Virologic Failures in Once-Daily Regimen of Abacavir, Lamivudine, and Tenofovir: 11th CROI, 2004; abstract 52. 
2. Jemsek J., Hutcherson P., Harper E. Poor Virologic Responses and Early Emergence of Resistance in Treatment Naive, HIV-infected Patients Receiving a Once Daily Triple Nucleoside Regimen of Didanosine, Lamivudine, and Tenofovir DF. 11th CROI, 2004; abstract 51 .

3. Podzamczer D., Ferrer E., Gatell J., et al. Early virological failure and occurrence of resistance in naive patients receiving tenofovir, didanosine and efavirenz. Antivir Ther 2004;9:S172.

4. Martinez E., Milinkovic A., de Lazzari E., et al. Pancreatic toxic effects associated with co-administration of didanosine and tenofovir in HIV-infected adults. Lancet. 2004;Jul 3;364(9428):65-7.

5. Negredo E., Molto J., Burger D., et al. Unexpected $\mathrm{CD}_{4}$ cell count decline in patients receiving didanosine and tenofovir-based regimens despite undetectable viral load. AIDS 2004;Feb 20,18(3):459-63. 